\title{
Evolutionary method of construction of solutions of polynomials and related generalized dynamics
}

\author{
Robert M. Yamaleev \\ Facultad de Estudios Superiores, \\ Universidad Nacional Autonoma de Mexico, \\ Cuautitlán Izcalli, Campo 1, C.P.54740, México. \\ Joint Institute for Nuclear Research, LIT, Dubna, Russia. \\ email: iamaleev@servidor.unam.mx
}

\begin{abstract}
Invariant theory as a study of properties of polynomials under translational transformations is developed. Class of polynomials with congruent set of eigenvalues is introduced. Evolution equations for eigenvalues and coefficients remaining the polynomial within proper class of polynomials are formulated. The connection with equations for hyper-elliptic Weierstrass and hyper-elliptic Jacobian functions is found. Algorithm of calculation of eigenvalues of the polynomials based on the evolution process is elaborated. Elements of the generalized dynamics with $n$-order characteristic polynomials are built.
\end{abstract}

\section{RESUMEN}

La teoría de invariantes es un estudio de las propiedades de los polinomios que se desarrolla en las transformaciones de traslación. Se introduce una clase de polinomios congruentes con un conjunto de valores propios. Se formulan ecuaciones de evolución de los valores propios y los coeficientes del polinomio restante dentro de la clase adecuada 
de los polinomios. Se encuentra la conexión con las ecuaciones de Weierstrass hiperelípticas y funciones jacobiano hiper-elíptica. Son elaborados algoritmos de cálculo de valores propios de los polinomios basado en el proceso de evolución.

Keywords: Nonspreading mapping, maximal monotone operator, inverse strongly-monotone mapping, fixed point, iteration procedure

Mathematics Subject Classification: 12Yxx

\section{Introduction}

The problem of construction of solutions of the polynomial equations as a certain functions of the coefficients is one of the oldest mathematical problems. E.Galois and H.Abel had proved that the polynomial higher than fourth order, in general, does not admit a presentation of solutions by radicals [3]. This rigorous mathematical theory directed mathematicians to look for the other ways of solutions. In particularly, the eigenvalues of the polynomials can be expressed in analytical way as certain functions of the coefficients [2], [7]. Ch.Hermite was first who found an elegant expression of eigenvalues of the quintic equation by modular functions [6]. The theory of elliptic functions originally was related with the problem of finding of eigenvalues of the cubic polynomial. In fact, the eigenvalues of the cubic equation admit presentation by Weierstrass elliptic functions at the periods [12]. It is clear, however, that for a search of analytical solutions of the $n>5$ degree polynomials one needs of mathematical tools beyond the elliptic functions. In this context as hopeful tools one may consider the theories of hyper-elliptic functions [9] and multi-complex algebras [8], [13].

The main purpose of the present paper is to construct an Algorithm for calculation roots of the polynomials. For that purpose, firstly, we study invariant properties of the polynomials with respect to simultaneous translations of the roots. Secondly, we build the system of evolution equations for the coefficients transforming the given polynomial into the polynomial with one trivial solution. The evolution process is directed in a such way that remain the original polynomial within proper class where the roots of the polynomials form congruent set of values. The coefficients of the polynomial with respect to the parameter of evolution are solutions of the Cauchy problem for ordinary differential equations. As soon as the Cauchy problem is resolved, the eigenvalues of initial polynomial are found simply by simultaneous translations of the set of eigenvalues of the final polynomial. Since the final polynomial possesses with one trivial solution, the degree of the polynomial is reduced from $n$ to $(n-1)$ which simplifies the process of solution. If solution of the obtained polynomial still is a difficult problem, then the method can be applied again in order to reduce the degree of the obtained polynomial. This process can be continued up till linear equation. The inverse process is fulfilled simply by simultaneous translations of the roots from the solution of the linear equation up till solutions of $n$-degree polynomial. It is shown, the evolution equations for the coefficients can be identified with equations for Weierstrass-type 
hyper-elliptic functions, whereas the evolution equations for the roots are given by equations for Jacobian-type hyper-elliptic functions. Furthermore, it is demonstrated a link between evolution equations for the coefficients of the polynomials and the classical dynamic equations. In $n$-order generalized mechanics the inner- and outer-momenta are inter-related as roots and coefficients of the characteristic $n$-degree polynomial. In particular $n=2$ case, we return to well-known equations of the relativistic dynamics closely related with quadratic polynomial of the mass-shell equation.

Besides the Introduction the paper contains the following sections.

In Section 2, the equations of evolution for the coefficients of $n$-degree polynomial are formulated. In Section 3, the Algorithm of finding of eigenvalues of the $n$-degree polynomial is built. In Section 4, some peculiarities of the cubic equation is explored. In Section 5, the elements of the relativistic dynamics based on quadratic characteristic polynomial is presented. In Section 6 elements of the generalized dynamics related with $n$-degree characteristic polynomial is outlined.

\section{Evolution equations for eigenvalues and coefficients of $n$ - degree polynomial}

If $F$ is a field and $q_{1}, \ldots, q_{n}$ are algebraically independent over $F$, the polynomial

$$
p(X)=\prod_{i=1}^{n}\left(X-q_{i}\right)
$$

is referred to as generic polynomial over $F$ of degree $n$. The polynomial equation of $n$-degree over field $F$ is written in the form

$$
p(X):=X^{n}+\sum_{k=1}^{n-1}(-)^{k}(n-k+1) P_{k} X^{n-k}+(-)^{n} P^{2}=0,
$$

where the coefficients $P_{k} \in F\left(q_{1}, \ldots, q_{n}\right)$. In this paper we shall restrict our attention only to polynomials with real coefficients and with simple roots. The signs at the coefficients in Eq.(2.1) are changed from term to term which allows in Vièta formulae to keep only the positive signs. The expressions at the coefficients are included for a convenience and have no real bearing on the theory. The mapping from the set of eigenvalues onto the set of coefficients is given by Vièta formulae

$$
\text { (a) } n P_{1}=\sum_{i=1}^{n} q_{i}, \quad \text { (b) } P^{2}=\prod_{i=1}^{n} q_{i}, \quad \text { (c) } P_{k}=\sum_{1 \leq r_{1}<\ldots<r_{k} \leq n} \prod_{j=1}^{k} q_{r_{j}}, k=2, \ldots, n-1,
$$

here $P_{1}, P_{k}, P^{2}$ are defined via elementary symmetric $k$-degree polynomials of the eigenvalues. The number of monomials inside $k$-th elementary polynomial is equal to binomial coefficient:

$$
C_{n}^{k}=\left(\begin{array}{c}
k \\
n
\end{array}\right)=\frac{n !}{k !(n-k) !} .
$$


Since the roots of the generic polynomial $p(X)$ are algebraically independent, this polynomial is, in some sense, the most general polynomial possible.

In $p(X)$ replace $X$ with $X=Y+P_{1}$. This transformation will eliminate the $(n-1)$-degree term, resulting in a polynomial of the form

$$
r(Y):=Y^{n}+\sum_{k=2}^{n-1}(-)^{k} R_{k} Y^{n-k}+(-)^{n} R_{0}=0 .
$$

The polynomials $p(X)$ and $r(Y)$ have the same splitting field and hence the same Galois group. Let $E$ be the splitting field of $r(Y)$, let $y_{k}, k=1, . ., n$ be its roots in $E$ and $G=G_{F}(E)$ be its Galois group.

\section{Lemma 2.1}

The coefficients $R_{k}, k=2, \ldots, n-1, R_{0}$ of Eq.(2.4) remain unaltered with respect to simultaneous translations of the eigenvalues of Eq.(2.1)

The Proof of the statement it follows from formula $Y=X-P_{1}$ which in terms of the eigenvalues is expressed as follows

$$
y_{k}=q_{k}-\frac{1}{n} \sum_{i=1}^{n} q_{i}=\frac{1}{n} \sum_{i \neq k}^{n}\left(q_{k}-q_{i}\right), k=1, \ldots, n .
$$

It is seen that the eigenvalues of Eq.(2.4) are represented by differences between the eigenvalues of Eq.(2.1), hence, they are invariants with respect to the simultaneous translations. Since the coefficients $R_{0}, R_{k}, k=2, \ldots, n-1$, are sum of uniform monomials of $y_{k}, k=1, \ldots, n$ they have same feature, namely, they are invariants with respect to simultaneous translations of the eigenvalues of Eq.(2.1).

\section{End of Proof.}

The polynomial $r(Y)$ we denominate as invariant polynomial (with respect to translations of the roots of $(2.1))$.

The main task of this section is to introduce evolution equations for the coefficients of Eq.(2.1) for which the coefficients of Eq.(2.4) $R_{k}, k=2, \ldots, n-1, R_{0}$ serve as the first integrals. This result is given by the following

\section{Theorem 2.2}

Let $q_{k}, k=1, \ldots, n$ be set of eigenvalues of polynomial equation of $n$-degree (2.1). Let differentials of all eigenvalues are equal to each other

$$
d q_{1}=d q_{2}=\ldots=d q_{k}=\ldots=d q_{n} .
$$

Then differentials of the coefficients satisfy the following system of equations:

$$
2 P_{n-1} d P_{1}=d P^{2},
$$




$$
d P_{n-k}=(k+2) P_{n-k-1} d P_{1}, \quad k=1, \ldots, n-2
$$

\section{Proof.}

From $(2.2 \mathrm{a})$ it follows

$$
d q_{k}=d P_{1}, \quad k=1, \ldots, n .
$$

Coefficients of the polynomial (2.1) are symmetric forms of the eigenvalues where $k$-th coefficient $(n-k+1) P_{k}$ consists of $C_{n}^{k}$ monomials of $k$-degree. Thus, the derivative of this coefficient $(n-k+$ 1) $d P_{k}$ contains $k C_{n}^{k}$ monomials and, since the derivatives of all eigenvalues are equal to $d P_{1}$, the derivative of $(n-k+1) P_{k}$ is proportional to $\lambda d P_{1}$ where the coefficient of proportionality consists of $k C_{n}^{k}(k-1)$-degree symmetric monomials. On the other hand, the symmetric polynomial of $(k-1)$-th degree can be expressed only by $C_{n}^{k-1}$ symmetric monomials of $(k-1)$-degree. This means the expression for $\lambda$ consists of $k C_{n}^{k} / C_{n}^{k-1}=n-k+1$ same symmetric polynomials of $(k-1)$-degree which are equal to the $(k-1)$-th coefficient $(n-k+2) P_{k-1}$. The result is expressed as follows

$$
(n-k+1) d P_{k}=(n-k+1)(n-k+2) P_{k-1} d P_{1}, \quad k=2,3, \ldots, n-1 .
$$

Differentiation of the last coefficient is obtained by direct calculation

$$
d P^{2}=2 P_{n-1} d P_{1},
$$

which completes the system of differential equations for the coefficients of Eq.(2.1).

\section{End of Proof.}

The following Lemma demonstrates an important role of the invariant polynomial in the evolution process.

\section{Lemma 2.3}

The first integrals of evolution equations (2.7)-(2.8) are given by coefficients of invariant polynomial (2.4).

\section{Proof}

In fact, the roots of Eq.(2.4), $y_{k}, k=1, \ldots, n$, according to formulae (2.5), are the first integrals of evolution equations for eigenvalues (2.6). Equations (2.7)-(2.8) are consequences of Eqs.(2.6), hence coefficients $R_{k}$ as algebraic functions of the solutions of evolution equations are first integrals of Eqs.(2.7)-(2.8),

\section{End of Proof}

Inversely by substituting $Y$ with $X-P_{1}$ in Eq.(2.4) we come back from invariant equation (2.4) to equation (2.1). Substitute $X-P_{1}$ instead of $Y$ and gather together powers of $X$, and the coefficients of the obtained polynomial compare with coefficients of Eq.(2.1). We shall see that the coefficients $P_{k}, k=1, \ldots, n-1$ now are expressed as $k$-degree polynomials of $P_{1}$ with coefficients consisting of $R_{k}, k=2, \ldots, n-1$. Especially notice, the invariant $R_{0}$ is defined by 
$n$-degree polynomial of $P_{1}$ the coefficients of which are certain polynomials of $R_{k} . k=2, \ldots, n-1$. The task is to define a explicit form of the polynomial of $P_{1}$. The general form of the desired polynomial can be presented as follows

$$
P_{1}^{n}+\sum_{k=2}^{n-1} P_{1}^{n-k} f_{k}\left(R_{1}, \ldots, R_{k}\right)+R_{0}=P^{2}
$$

Now, the next task is to find an explicit form of the function $f_{k}$. For that purpose explore firstly the case $P^{2}=0$. In this case one of the solutions of Eq.(2.1) is equal to zero. Then the corresponding solution of the invariant polynomial is

$$
y_{1}=-P_{1}\left(P^{2}=0\right)=-P_{1}(0)
$$

which satisfy Eq.(2.4). By replacing $Y$ with $\left(-P_{1}(0)\right)$ in Eq.(2.4) we come to the following equation for $P_{1}(0)$ :

$$
P_{1}^{n}(0)+\sum_{k=2}^{n-1} R_{k} P_{1}^{n-k}(0)+R_{0}=0
$$

Notice, here the signs at all coefficients are positive. Since we use evolution equations (2.7)-(2.8) in order to pass from Eq.(2.1) with coefficients $\left\{P_{k}, P^{2} \neq 0\right\}$ to the polynomial with coefficients $\left\{\widetilde{P}_{k}, P^{2}=0\right\}$, these two polynomials possess with the same invariants as the original one. Hence, when $P^{2} \neq 0$ equation (2.10) is transformed as follows

$$
P_{1}^{n}+\sum_{k=2}^{n-1} R_{k} P_{1}^{n-k}+R_{0}=P^{2}
$$

Notice, the situation is somewhat similar the conventional Classical Invariant Theory of Polynomials [10]. If classical invariant theory is a study of properties of a polynomial $p(x)$ that are unchanged under fractional linear transformations, within the framework of the present approach we study properties of polynomials under translational transformations.

\section{Theorem 2.4}

If evolution of the coefficients of Eq.(2.1) obey equations (2.7)-(2.8), then

$$
(k+1) P_{n-k}=\frac{1}{k !} \frac{d^{k}}{d P_{1}^{k}} P^{2} .
$$

\section{Proof.}

Differentiate equation (2.11) by taking into account that $R_{0}, R_{k}, k=1, \ldots, n-1$ are constants. We get

$$
d P_{1}\left(n P_{1}^{n-1}+\sum_{k=2}^{n-2}(n-k) R_{k} P_{1}^{n-k-1}+R_{n-1}\right)=d P^{2} .
$$


Compare this equation with Eq.(2.9). It is seen, the expression inside brackets at $d P_{1}$ is nothing else than the coefficient $2 P_{n-1}$ expressed as a polynomial of $P_{1}$ with invariant coefficients. Hence,

$$
2 P_{n-1}=n P_{1}^{n-1}+\sum_{k=2}^{n-2}(n-k) R_{k} P_{1}^{n-k-1}+R_{n-1}=\frac{d}{d P_{1}} P^{2}
$$

Next, differentiate (2.14) with respect to $P_{1}$, this leads to the equation

$$
d P_{n-1}=3 P_{n-2} d P_{1},
$$

where $3 P_{n-2}$ is the following coefficient of Eq.(2.1):

$$
3 P_{n-2}=\frac{n(n-1)}{2} P_{1}^{n-2}+\sum_{k=2}^{n-3} \frac{(n-k)(n-k-1)}{2} R_{k} P_{1}^{n-k-2}+R_{n-2} .
$$

Consequently,

$$
3 P_{n-2}=\frac{d^{2}}{d P_{1}^{2}} P^{2}
$$

The next step of this procedure leads to the equation

$$
d P_{n-2}=4 P_{n-3} d P_{1}
$$

where

$$
4 P_{n-3}=\frac{n(n-1)(n-2)}{1 \cdot 2 \cdot 3} P_{1}^{n-3}+\sum_{k=2}^{n-4} \frac{(n-k)(n-k-1)(n-k-2)}{1 \cdot 2 \cdot 3} R_{k} P_{1}^{n-k-3}+R_{n-3} .
$$

Hence,

$$
4 P_{n-3}=\frac{d^{3}}{d P_{1}^{3}} P^{2} .
$$

By induction we come to the general formula for $l$-th coefficient $P_{n-l}$ :

$$
(l+1) P_{n-l}=\left(\begin{array}{c}
l \\
n
\end{array}\right) P_{1}^{n-l}+\sum_{k=2}^{n-l-1}\left(\begin{array}{c}
l \\
n-k
\end{array}\right) R_{k} P_{1}^{n-k-l}+R_{n-l}=\frac{1}{l !} \frac{d^{l}}{d P_{1}^{l}} P^{2} .
$$

\section{End of Proof.}

This theorem has some interesting consequences.

\section{Corollary 2.5}

The following representation for polynomial $p(X)$ holds true

$$
p(X)=\exp \left(-X \frac{d}{d P_{1}}\right) P^{2}=0 .
$$

\section{Proof}


The generator of translation defined by Euler operator is represented by the following expansion

$$
\exp \left(-X \frac{d}{d P_{1}}\right)=1-X \frac{d}{d P_{1}}+X^{2} \frac{1}{2 !} \frac{d^{2}}{d P_{1}^{2}}+\ldots+X^{n} \frac{1}{n !} \frac{d}{d P_{1}^{n}}+\ldots .
$$

By differentiating Eq.(2.11) $n$-times with respect to $P_{1}$, we get

$$
\frac{d}{d P_{1}^{n}} P^{2}=n !
$$

Hence the sum in (2.20) is completed by this term. Thus,

$$
\exp \left(-X \frac{d}{d P_{1}}\right) P^{2}=p(X)
$$

Here the variable $X$ means one of the roots of Eq.(2.1) in quality of which let us take $q_{n}$.

The derivative with respect to $P_{1}$ due to (2.6) can be expressed by the following sum

$$
\frac{d}{d P_{1}}=\sum_{k=1}^{n} \frac{\partial q_{k}}{\partial P_{1}} \frac{\partial}{\partial q_{k}}=\sum_{k=1}^{n} \frac{\partial}{\partial q_{k}}
$$

Then,

$$
\exp \left(-q_{n} \frac{d}{d P_{1}}\right) P^{2}=\prod_{k=1}^{n-1} \exp \left(-q_{n} \frac{\partial}{\partial q_{k}}\right) \exp \left(-q_{n} \frac{\partial}{\partial q_{n}}\right) .
$$

Now, take into account Vièta formula for $P^{2}$ given by (2.2). The Euler operator (2.24) will translate each root by $q_{n}$. The last operator in (2.24) acts only upon $n$-th root resulting $q_{n}-q_{n}=0$. Hence,

$$
\exp \left(-X \frac{d}{d P_{1}}\right) P^{2}=p(X)=0 .
$$

\section{End of Proof.}

\section{Algorithm of finding the eigenvalues of $n$-degree polyno- mials}

In the previous section we have found that evolution governed by equations (2.7)-(2.8) remains the polynomials within certain class of polynomials with congruent set of eigenvalues. This fact prompts an idea to use the evolution equations in order to come to the simplified polynomial. The main purpose of this section is to elaborate an algorithm of solution of $n$-degree polynomial equation by reducing the degree of the polynomial equation. The evolution from original polynomial to the simplified polynomial is fulfilled in such a way which remains unaltered coefficients of the invariant polynomial. Hence, initial and final polynomials of this evolution process will possess with congruent set of eigenvalues, so that the solutions of the former can be obtained from the solutions of the latter by simultaneous transformations of translation. 
The algorithm is directed in a such way that at the final point of evolution we shall come to the polynomial with $P^{2}=0$. The last coefficient $P^{2}$ is used as a parameter of the evolution. This evolution will remain unaltered the coefficients of Eq.(2.4), hence from solutions of the polynomial with $P^{2}=0$ we may come to the solutions of the original equation simply by translation of the set of solutions of the simplified polynomial with new coefficients.

Denote $x=P^{2}$. Re-write Eq.(2.8) with respect to $x$. We get

$$
\begin{gathered}
2 P_{n-1} d P_{1}=d x, \\
\frac{d P_{n-k}}{d x}=(k+2) P_{n-k-1} \frac{d P_{1}}{d x}, \quad k=1, \ldots, n-3 ; \\
\frac{d P_{2}}{d x}=n P_{1} \frac{d P_{1}}{d x} .
\end{gathered}
$$

This is a Cauchy problem with initial data $P_{k}\left(x=P^{2}\right), \quad k=1,2,3, \ldots, n-1$. The variable $x$ runs from $x=P^{2}$ up till $x=0$. These equations usually are resolved by using the Cauchy-Lipschitz method of calculation [4]. The procedure is fulfilled by dividing the interval $\left(x_{0}, 0\right)$ into $N$ parts:

$$
\Delta x_{0}=x_{1}-x_{0}, \Delta x_{i}=x_{i+1}-x_{i}, \Delta x_{n-1}=x-x_{n-1},
$$

where $x_{i}<x_{i+1}, x_{N}=0$.

In this way the continuous evolution process is transformed into discrete process consisting of $N$ steps. At the last step of discrete evolution process we come to $n$-degree polynomial free of the last coefficient:

$$
\stackrel{(1)}{X^{n}}+\sum_{k=1}^{n-1}(-)^{k}(n-k+1) \stackrel{(1)}{P_{k}} X^{k}=0 .
$$

One of the solutions is trivial, excluding this solution we come to the polynomial of $(n-1)$-degree:

$$
X^{n-1}+\sum_{k=1}^{n-1}(-)^{k}(n-k+1) \stackrel{(1)}{P_{k}} X^{k}+2 P_{n-1}^{(1)}=0 .
$$

Let us mention that Eq.(3.4) possesses with same invariants as original one, i.e. Eq.(2.1). Suppose that the solutions of Eq.(3.5) are $\stackrel{(1)}{q}_{k}, k=1, \ldots, n-1$. Complete this set of solutions by $\stackrel{(1)}{q}_{n}=0$. Then the solutions of original equation one may find simply by using the following translations

$$
q_{k}=\stackrel{(1)}{q_{k}}+P_{1}-\stackrel{(1)}{P_{1}}, k=1, \ldots, n .
$$

If solution of the $(n-1)$-degree equation still is difficult problem, then one may apply the algorithm again in order to reduce the problem of solution of $(n-1)$-degree equation to the problem of solution $(n-2)$-degree equation. This process can be continued up till quadratic or linear equation. At each step of the iteration one will find an information on coefficient $\stackrel{(r)}{P_{1}}$. At the $r$-th iteration one deals with $(n-r)$-degree equation in the form

$$
X^{n-r}+\sum_{k=1}^{(r)}(-)^{k}(n-k+1) \stackrel{(r)}{P_{k}} X^{k}+(r+1) P_{n-r}^{(r)}=0 .
$$


At this stage the norm of the coefficient $\stackrel{(r)}{P}_{n-r}$ will fulfil the role of evolution parameter of the next evolution process. As soon as the solutions of the lowest polynomial is found, the inverse process of iterations will consist of translations of the known set of eigenvalues with known parameter of translation. Let the last step of iteration be a linear equation with unique solution:

$$
\stackrel{(n-1)}{q_{1}}=n \stackrel{(n-1)}{P_{1}}
$$

Then the solutions of the original equation (2.1) are found as a result of the following set of translations

$$
\begin{gathered}
q_{1}=n \stackrel{(n-1)}{P_{1}}+n \sum_{r=2}^{n} \frac{1}{r}\left(\begin{array}{c}
(n-r) \\
P_{1}
\end{array}-\begin{array}{c}
(n-r+1) \\
P_{1}
\end{array}\right), \\
q_{s}=\sum_{r=s}^{n} \frac{1}{r}\left(\begin{array}{c}
(n-r) \\
P_{1}
\end{array}-\stackrel{(n-r+1)}{P_{1}}\right), s=2,3, \ldots, n ;
\end{gathered}
$$

where $\stackrel{(0)}{P_{1}}=P_{1}$.

\section{Relativistic Lorentz-force equations and related quadratic polynomial}

There exist an certain inter-relation between the evolution of the polynomials and the dynamic systems. The aim of this section is to demonstrate as evolution of the quadratic polynomial is related with the relativistic Lorentz-force equations. In the sequel, we shall use this example as a starting platform to pass to case of higher degree polynomials and related generalized dynamics. Let us start with generic quadratic polynomial

$$
X^{2}-2 P_{0} X+P^{2}=0
$$

with real coefficients $P_{0}^{2} \geq P^{2}$, and real eigenvalues $p_{1}^{2}, p_{2}^{2}$. This polynomial closely related with the relativistic dynamics [14], [5]. In order to demonstrate this fact let us start from the Lorentz-force equations describing a motion of the charged particle inside external electromagnetic fields $\mathbf{E}, \mathbf{B}^{1}$

$$
\begin{gathered}
\frac{d \mathbf{P}}{d \tau}=\left(\mathbf{E} P_{0}+[\mathbf{P} \times \mathbf{B}]\right), \frac{d P_{0}}{d \tau}=(\mathbf{E} \cdot \mathbf{P}), \\
\frac{d \mathbf{r}}{d \tau}=\mathbf{P}, \quad \frac{d t}{d \tau}=P_{0} .
\end{gathered}
$$

Consider projection of equations (4.2) on direction of the motion defined by unit vector $\vec{n}=\mathbf{P} / P$ :

$$
\frac{d P}{d \tau}=(\mathbf{E} \cdot \mathrm{n}) P_{0}, \frac{d P_{0}}{d \tau}=(\mathbf{E} \cdot \mathrm{n}) P
$$

\footnotetext{
${ }^{1}$ Hereafter for the sake of simplicity we omit all parameters like charge, mass, light-velocity and other parameters regularizing physical dimensions taking them equal to unit.
} 
Then one deals only with the lengths of the momenta $P_{0}, P$. Here parameter of evolution $\tau$ means a proper time, simplify Eqs.(4.4) by introducing a new evolution parameter by

$$
\frac{d P}{d s}=P_{0}, \quad \frac{d P_{0}}{d s}=P,
$$

where

$$
\frac{d s}{d \tau}=(\mathbf{E} \cdot \mathrm{n}) .
$$

The first constant of motion is easily found

$$
P_{0}^{2}-P^{2}=M^{2}
$$

Here $M^{2}$ is a constant of motion. Conventionally, this constant is interpreted as a square of inertial mass [11]. Inside stationary potential field, when $\mathbf{E}=-\nabla V(r)$, dynamic equations imply the other integral of motion, the energy,

$$
\mathcal{E}_{0}=P_{0}+V(r) .
$$

At the rest state where $P=0$ one obtains $P_{0}(P=0)=M$. Consider the following linear combinations of two kinds of energies:

$$
p_{1}^{2}=P_{0}-M, \quad p_{2}^{2}=P_{0}+M .
$$

From formulae (4.7) and (4.9) it follows

$$
P=p_{1} p_{2}, P_{0}=\frac{1}{2}\left(p_{2}^{2}+p_{1}^{2}\right), \quad M=\frac{1}{2}\left(p_{2}^{2}-p_{1}^{2}\right) .
$$

Notice, the first two formulae of (4.10) mean Vièta formulae for quadratic polynomial equation (4.1). Substitution $X=Y+P_{0}$ in (4.1) leads to invariant equation

$$
Y^{2}=P_{0}^{2}-P^{2}=M^{2},
$$

the invariant coefficient of which is equal to the invariant of physical motion. Quadratic equation for $P_{0}$ is given by

$$
P_{0}^{2}-M^{2}=P^{2} .
$$

Differentiating this equation we derive evolution equations for the coefficients, which in the case of quadratic polynomial, of course, is a simple task:

$$
2 P_{0} P=\frac{d}{d s} P^{2}, \quad \frac{d}{d s} P_{0}=P .
$$

Solutions of these evolution equations are given by hyperbolic cosine-sine functions

$$
P=M \sinh (s), \quad P_{0}=M \cosh (s) .
$$

Then the eigenvalues of Eq.(5.1) are expressed by hyperbolic cosine-sine functions of one-half argument

$$
p_{1}^{2}=\sqrt{2 M} \sinh \left(\frac{s}{2}\right), \quad p_{2}^{2}=\sqrt{2 M} \cosh \left(\frac{s}{2}\right) .
$$


Now, consider so-called the effective potential representation. With this purpose let us come back to the equations written with respect to proper-time $\tau$. Then from the second equation of (4.13) it follows

$$
\mathcal{E}_{0}=P_{0}+V(r)
$$

Further, replace $P_{0}$ by $\mathcal{E}_{0}-V(r)$ in the first equation of (4.13), this gives

$$
\frac{d}{d s} \mathbf{P}=-\nabla V(r)\left(\mathcal{E}_{0}-V(r)=-\nabla W\left(r, \mathcal{E}_{0}\right),\right.
$$

where the effective potential is defined by

$$
W\left(r \mathcal{E}_{0}\right)=\mathcal{E}_{0} V(r)-\frac{1}{2} V^{2}(r)
$$

Eq.(4.16) is the Newtonian equation of motion with effective potential $W$ and with the energy

$$
\mathcal{E}=\frac{1}{2} P^{2}+W\left(r, \mathcal{E}_{0}\right)=\frac{1}{2}\left(\mathcal{E}_{0}^{2}-M^{2}\right) .
$$

\section{Cubic polynomial equation and related dynamics}

In the previous section we have demonstrated as the relativistic dynamics is related with the evolution of quadratic polynomial. This example provides us with appropriate tool in order to construct generalized scheme based on the evolution of polynomials of higher order. In this section we explore the case of cubic polynomial

$$
p(X)=X^{3}-3 P_{1} X^{2}+2 P_{2} X-P^{2}=0,
$$

where coefficients and eigenvalues are connected by the following formulae

$$
3 P_{1}=q_{1}+q_{2}+q_{3}, 2 P_{2}=q_{1} q_{2}+q_{2} q_{3}+q_{3} q_{1}, P^{2}=q_{1} q_{2} q_{3}
$$

Let us assume that $p(X)$ is irreducible. By replacing $X$ with $X=Y+P_{1}$ we come to invariant polynomial

$$
Y^{3}+R_{2} Y-R_{0}=0
$$

where

$$
\text { (a) } R_{2}=2 P_{2}-3 P_{1}^{2}, \quad \text { (b) } P_{1}^{3}+R_{2} P_{1}+R_{0}=P^{2} \text {. }
$$

The eigenvalues and the coefficients of this polynomial are invariants with respect to simultaneous translations of the eigenvalues of Eq. (5.1). Obviously this statement is a consequence of the formula $Y=X-P_{1}$ from which it follows

$$
3 y_{1}=e_{2}-e_{3}, 3 y_{2}=e_{3}-e_{1}, 3 y_{3}=e_{1}-e_{2},
$$

where

$$
e_{1}=q_{3}-q_{2}, e_{2}=q_{1}-q_{3}, e_{3}=q_{2}-q_{1} \text {. }
$$


Evolution equations remaining unaltered coefficients $R_{0}, R_{2}$ are obtained directly by differentiating Eqs.(5.4). We get

$$
\begin{gathered}
d P_{1}\left(3 P_{1}^{2}+R_{2}\right)=2 P_{2} d P_{1}=d P^{2}, \\
d P_{2}=3 d P_{1} .
\end{gathered}
$$

The following equations for the eigenvalues hold true

$$
\frac{d}{d s} q_{k}=\frac{d}{d s} P_{1}=P, k=1,2,3 .
$$

The generalized dynamics of third order is an object of special interest because this dynamics is closely related with the classical elliptic functions. The solutions of the evolution equations for the eigenvalues and the coefficients of the cubic polynomial can be represented via elliptic Jacobi and Weierstrass functions, correspondingly [1]. On making use (5.7) from (5.4b) we come to the following differential equation

$$
P_{1}^{3}+R_{1} P_{1}+R_{0}=\left(\frac{d P_{1}}{d s}\right)^{2} .
$$

Write this equation in the following designations

$$
\left(\frac{d \wp}{d z}\right)^{2}=4 \wp(z)^{3}-g_{2} \wp(z)-g_{3},
$$

where $z=4 s, g_{2}=-4 R_{1}, \quad g_{3}=-4 R_{0}$ and $\wp(2 s)=P_{1}(s)$. The integral formula for $\wp(z)$ is given by

$$
z=\int_{\wp}^{\infty}\left(4 x^{3}-g_{2} x-g_{3}\right)^{-1 / 2} d x .
$$

The functions $\wp(z)$ and $\wp^{\prime}(z)$ are Weierstrass elliptic functions with periods $2 \omega_{1}, 2 \omega_{2}$. Define $\omega_{3}=-\omega_{1}-\omega_{2}$. Then the values

$$
\wp\left(\omega_{1}\right), \wp\left(\omega_{2}\right), \wp\left(\omega_{3}\right)
$$

are the roots of cubic equation

$$
4 x^{3}-g_{2} x-g_{3}=0 .
$$

Introduce new variables $p_{k}, k=1,2,3$ by $p_{k}^{2}=q_{k}$. Then $P=p_{1} p_{2} p_{3}$. For $p_{k}$ evolution equations are derived from (5.7):

$$
\frac{d p_{1}}{d s}=p_{2} p_{3}, \quad \frac{d p_{2}}{d s}=p_{1} p_{3}, \quad \frac{d p_{3}}{d s}=p_{2} p_{1} .
$$

Solutions of these equations are given by quotients of Jacobi elliptic functions. Let $\operatorname{sn}(u), c n(u), d n(u)$ be a set of Jacobi elliptic functions. Define quotients of these functions (in Glaisher notations [1]) by

$$
n s=-\frac{1}{s n}, \quad c s=-\frac{c n}{s n}, \quad d s=-\frac{d n}{s n},
$$

which obey the following differential equations

$$
\frac{d}{d u} n s(u)=c s(u) d s(u), \frac{d}{d u} c s(u)=n s(u) d s(u), \frac{d}{d u} d s(u)=c s(u) n s(u),
$$


with

$$
n s^{2}-c s^{2}=1, d s^{2}-c s^{2}=1-\mathrm{k}^{2} .
$$

From Eqs.(5.12) it follows that the values

$$
e_{1}=p_{3}^{2}-p_{2}^{2}, e_{2}=p_{1}^{2}-p_{3}^{2},
$$

are constants of motion. Hence, the solutions of Eqs.(5.12) are proportional to the Jacobi elliptic functions

$$
p_{3}=\sqrt{e_{1}} n s(u, \mathrm{k}), p_{2}=\sqrt{e_{1}} c s(u, \mathrm{k}), p_{1}=\sqrt{e_{1}} d s(u, \mathrm{k}), \mathrm{k}^{2}=1-\frac{e_{2}}{e_{1}} .
$$

The final result is formulated by the following

\section{Statement:}

If the evolution of squared roots of the eigenvalues of the cubic equation obey equations for Jacobi elliptic functions, then the evolution of the coefficients are governed by equations for Weierstrass elliptic functions.

For the cubic equation there exist also another possibility to express its eigenvalues, namely, by using trigonometric functions. The eigenvalues of the invariant polynomial (5.3) are given by formulae:

$$
y_{1}=-\frac{2}{3} \sqrt{3 R_{1}} \cos \theta, y_{2}=\frac{1}{3} \sqrt{3 R_{1}}(\cos \theta+\sqrt{3} \sin \theta), y_{3}=\frac{1}{3} \sqrt{3 R_{1}}(\cos \theta-\sqrt{3} \sin \theta) .
$$

It is easy to verify that

$$
y_{1}+y_{2}+y_{3}=0, \quad y_{1} y_{2}+y_{2} y_{3}+y_{3} y_{1}=-R_{1} .
$$

Equation for the last coefficient, $R_{0}$, leads to trigonometric equation for $\cos (\theta)$ :

$$
\begin{gathered}
-R_{0}=y_{1} y_{2} y_{3} \\
=\cos \theta\left(\cos ^{2} \theta-3 \sin ^{2} \theta\right)=4 \cos ^{3} \theta-3 \cos \theta=\frac{27}{2} \frac{R_{0}}{\left(\sqrt{3 R_{1}}\right)^{3}} .
\end{gathered}
$$

By using the trigonometric formula

$$
\cos (3 \theta)=4 \cos ^{3} \theta-3 \cos \theta
$$

we come to simple trigonometric equation:

$$
\cos (3 \theta)=\frac{3 \sqrt{3}}{2} \frac{R_{0}}{\sqrt{R_{1}^{3}}} .
$$

This equation is valid if only if the cubic equation possesses with real solutions which provided by the following inequality

$$
\left(\frac{R_{0}}{2}\right)^{2}<\left(\frac{R_{1}}{3}\right)^{3} .
$$


The algorithm elaborated in the previous section in the case of cubic equation (5.1) is simplified as follows:

$$
2 P_{2} d P_{1}=2 P_{2} d x, \quad 2 P_{2} d P_{2}=3 P_{1} d x,
$$

with the initial data $P_{1}\left(x=P^{2}\right)=P_{1}, P_{2}\left(x=P^{2}\right)=P_{2}$. At the final stage when $x=P^{2}=0$ quantities $P_{1}\left(P^{2}=0\right)=\stackrel{(1)}{P_{1}}, P_{2}\left(P^{2}=0\right)=\stackrel{(1)}{P_{2}}$ satisfy the following relationships

$$
R_{2}=2 \stackrel{(1)}{P_{2}}-3 \stackrel{(1)}{P_{1}^{2}}, \quad \stackrel{(1)}{P_{1}^{3}}+R_{1} \stackrel{(1)}{P_{1}}+R_{0}=0 .
$$

Thus the new equation possesses with same invariants as the original one,

$$
X^{3}(0)-3 \stackrel{(1)}{P} X_{1}^{2}(0)+2 \stackrel{(1)}{P_{2}} X(0)=0 .
$$

Equations (5.1) and (5.19) possess with congruent eigenvalues where, notice, Eq.(5.19) has one trivial solution. Therefore the problem is reduced to the problem of solution of quadratic equation

$$
X^{2}(0)-3 \stackrel{(1)}{P} X_{1}^{2}(0)+2 \stackrel{(1)}{P_{2}} X(0)=0 .
$$

From solutions of Eq.(5.20) to the solutions of Eq.(5.1) one comes simply by the set of translations:

$$
q_{1}=P_{1}-\stackrel{(1)}{P_{1}}, \quad q_{2}=q_{2}(0)+P_{1}-\stackrel{(1)}{P_{1}}, \quad q_{3}=q_{3}(0)+P_{1}-\stackrel{(1)}{P_{1}} .
$$

The dynamics related with the cubic polynomial, evidently, is characterized with two first constants of motion $R_{2}, R_{0}$ which do not depend of potential field. In quality of evolution equations we have to use Eqs.(5.6)-(5.7) written with respect to parameter of evolution defined in (4.6):

$$
P_{2}=\frac{d}{d s} P, \quad \frac{d}{d s} P_{2}=3 d P_{1}, \quad \frac{d}{d s} P_{1}=P .
$$

Evolution equations for the eigenvalues, correspondingly, are given by

$$
\frac{d}{d s} p_{k}^{2}=P, \quad k=1,2,3
$$

From these equations it follows that the constants of motion are given by formulae

$$
M_{1}=p_{2}^{2}-p_{3}^{2}, M_{2}=p_{3}^{2}-p_{1}^{2}, M_{3}=p_{1}^{2}-p_{2}^{2}
$$

In order to include into this scheme the potential field $V(r)$ we have to use equation (4.6):

$$
\frac{d s}{d \tau}=(\mathrm{U} \cdot \mathrm{n}), \quad \mathrm{U}=\nabla V(r), \quad P \mathrm{n}=\mathbf{P} .
$$

Furthermore, the set of evolution equations has to be completed with the interrelation between momentum and velocity with respect to time-like parameter:

$$
\mathbf{P}=\frac{d \mathbf{r}}{d \tau}
$$


In these designations evolution equations (5.21) are transformed into the following dynamic equations

$$
\frac{d}{d \tau} \mathbf{P}=-\nabla V(r) P_{2}, \quad \frac{d}{d \tau} P_{2}=-3(\mathbf{P} \cdot \nabla) V(r) P_{1}, \quad \frac{d}{d \tau} P_{1}=-(\mathbf{P} \cdot \nabla) V(r) .
$$

In the case of stationary potential, beside the first integrals $R_{2}, R_{0}$, the equations imply another integral of motion, the energy

$$
\mathcal{E}_{1}=P_{1}+V
$$

By substituting the expression for $P_{1}$ from (5.27) into the second equation of Eqs.(5.26), we get

$$
\mathcal{E}_{2}=P_{2}+3 \mathcal{E}_{1} V-\frac{3}{2} V^{2}=\frac{1}{2}\left(R_{2}+3 \mathcal{E}_{1}^{2}\right) .
$$

This is second expression for the energy. Next, express from this formula $P_{2}$ and substitute into first equation of (5.26). This leads to Newtonian equation

$$
\frac{d}{d \tau} \mathbf{P}=-\nabla W\left(r, \mathcal{E}_{1}\right)
$$

with effective potential

$$
2 W\left(r, \mathcal{E}_{1}\right)=\mathcal{E}_{2} V(r)-3 \mathcal{E}_{1} V^{2}(r)+V^{3}(r) .
$$

From Eq.(5.29) one may find formula for the total energy

$$
\mathcal{E}=\frac{1}{2} P^{2}+W=\frac{1}{2}\left(R_{0}+R_{2} \mathcal{E}_{1}+\mathcal{E}_{1}^{3}\right)
$$

\section{Generalized dynamics related with evolution of $n$-degree polynomial}

Now we have accumulated enough experience in order to be able to build a general form of the dynamics related with $n$-degree polynomials. Let us start with evolution equations (2.8) written for $n$-degree polynomial (2.1). Dynamic equations with respect to some time-like evolution parameter $\tau$ describing a motion inside stationary potential field $V(r)$ are formulated in the following form [16]:

$$
\begin{gathered}
\frac{d \mathbf{P}}{d \tau}=\mathrm{U} P_{n-1}, \\
\frac{d P_{k}}{d \tau}=(\mathrm{U} \cdot \mathbf{P}) P_{k-1}(n-k+2), \quad k=2, \ldots, n-1, \\
\frac{d P_{1}}{d \tau}=(\mathrm{U} \cdot \mathbf{P}) .
\end{gathered}
$$

The set of evolution equations has to be completed with the interrelation between momentum and velocity given by Eq.(5.25). This system of dynamic equations remain invariant the coefficients $R_{0}, R_{k}, k=1, \ldots, n-1$. The motion of a physical system obeying to these equations possesses with 
the set of inner and outer momenta. Evolution of the set of outer-momenta $\left\{P^{2}, P_{k}, k=1, \ldots, n-1\right\}$ are given by Eqs.(6.1a,b,c), whereas the evolution of the inner-momenta $\left\{p_{k}, k=1, \ldots, n\right\}$ obey

$$
\frac{d p_{k}^{2}}{d \tau}=(\mathrm{U} \cdot \mathbf{P}), k=1, \ldots, n .
$$

The first integrals of this system are given by

$$
M_{i k}=p_{i}^{2}-p_{k}^{2}, \quad i \neq k .
$$

From Eq.(6.1c) we find the first constant of integration, the energy $\mathcal{E}_{1}=P_{1}+V$, or $P_{1}=\mathcal{E}_{1}-V$. By substituting $P_{1}$ into $k=2$-th equation of (6.1b) we find the other constant of integration (the second expression for the energy):

$$
\mathcal{E}_{2}=P_{2}+n E_{1} V-\frac{n}{2} V^{2} .
$$

Next, by substituting $P_{2}$ from the last expression into the following equation with $k=3$ we shall find the third constant of integration:

$$
\mathcal{E}_{3}=P_{3}-(n-1) E_{2} V+\frac{n(n-1)}{2} E_{1} V^{2}-\frac{n(n-1)}{2 \cdot 3} V^{3} .
$$

Continue this process up till $(n-1)$-th stage. Then, at $(n-1)$-th stage we shall find the expression for $P_{n-1}$. By using this formula in Eq.(6.1a) we come to Newtonian equation

$$
\frac{d}{d \tau} \mathbf{P}=-\nabla W\left(r, \mathcal{E}_{1}\right),
$$

where the effective potential is given by the following series

$$
W\left(r, \mathcal{E}_{1}\right)=\frac{1}{2} \sum_{k=1}^{n}(k+1) \mathcal{E}_{n-k} V^{k}(r)(-1)^{k+1}, \quad \text { with } \mathcal{E}_{0}=\frac{1}{n+1} .
$$

From Eq.(6.4) it follows formula for the total energy

$$
\mathcal{E}=\frac{1}{2} P^{2}+W=\frac{1}{2}\left(R_{0}+\sum_{k=2}^{n-1} R_{k} \mathcal{E}_{1}^{k-1}+\mathcal{E}_{1}^{n}\right) .
$$

\section{Concluding remarks}

This is a prodigious fact that the evolution equations elaborated for polynomials serve as dynamic equations for the generalized dynamics. Notice, the Algorithm of calculation of eigenvalues of the polynomials elaborated in this paper is distinct of the numerical methods of calculations which principally are based on iteration process with initial sampling, or tentative data of solutions, so that effectiveness of these algorithms depends of the initial data. The iteration process based on the present Algorithm uses in the quality of initial data the coefficients of the original polynomial. The method elaborated here can be considered also as a theory of functional connection between 
coefficients and eigenvalues of the polynomial expressed as one-valued Weierstrass-type and Jacobitype hyper-elliptic functions. Evidently, the present method without any principal difficulties can be continued to the case of polynomials defined over the field of complex numbers.

We have restricted our attention only with the dynamic equations over one-dimensional coordinate space. On the theory of the generalized dynamics in $4 D$ space with physical units one may consult in Refs.[15] and [16] and the references therein.

Received: February 2009. Revised: September 2009.

\section{References}

[1] Akhiezer N.I., Elements of theory of elliptic functions, Moscow, "Nauka", 1970.

[2] G.Belardinelli, Fonctions hypergeometriques de plusieurs variables et resolution analitique des equations algebriques generales. Paris: Gauthier-Villars, 1960.

[3] F.Cajoris, History of Mathematics, New York, 1919, pp.349-350.

I.Richards, An application of Galois theory to elementary arithmetic, Advances in Mathematics 13 (1974) 268-273.

[4] Harold T.Davis, Introduction to nonlinear differential and integral euations. Dover Publications, INC.,N.Y., 1968. (ISBN 0-486-60971-5)

[5] P.Fjelstad, S.Gal, Two dimensional geometries, topologies, trigonometries and physics generated by complex-type numbers, Advances in Applied Clifford algebras, 11(1), (2001), 81.

[6] Ch.Hermite, Sur la resolution de l'eqation du cinquieme degree C.R.Acad.Sci.Paris. - 1858. V.46.

Ch.Hermite, Sur l'eqation du cinquieme degree C.R.Acad.Sci.Paris. - 1865. V.46; 1866.-V.62.

[7] L.K.Lakhtin, Algebraic equations resolvables in hypergeometric functions (in russian) M.,1893. Differential resolvents of algebraic equations of higher degree. M.,1896.

[8] L.N.Lipatov, M.Raush de Traubenberg, G.G.Volkov, On ternary complex analysis and its applications. arXiv: 0711.0809v1 [math-ph ] 6 Nov. 2007.

[9] L.J.Mordell, On the rational solutions of the indeterminate equations of the 3-rd and 4-th degrees. Proc.Camb.Phill.Soc. 21 (1922) 179-192.

[10] P.J.Olver, Classical Invariant Theory, LondonMathematical Society Student Texts 44, Cambridge Univeristy Press, 1999.

[11] A.R.Rodriguez-Dominguez, Lorentz.force equations as Heisenberg equations for 4D-quantum system. Revista Mexicana de Fisica 53 (4) (2007) 270-280. 
[12] H.Weber, Lehrbuch der Algebra.Bd.2. Gruppen. Lineare Gruppen. Anwendungen der Gruppen Theorie. Algebraische Zahlen.- Braunschweig, 1899.

H.Weber, Lehrbuch der Algebra.Bd.3. Elliptische Functionen und algebraische Zahlen.- Braunschweig, 1908.

[13] R.M.Yamaleev, Multicomplex algebras on polynomials and generalized Hamilton dynamics, J.Math.Anal.Appl., 322, (2006), 815-824.

R.M.Yamaleev, Complex algebras on N-order polynomials and generalizations of trigonometry, oscillator model and Hamilton dynamics. Advances in Applied Clifford algebras, 15(1), (2005), 123.

[14] R.M.Yamaleev, "Relativistic Equations of Motion within Nambu's Formalism of Dynamics", Ann.Phys. 285 (2) (2000) 141-160.

[15] R.M.Yamaleev, "Generalized Newtonian Equations of Motion", Ann.Phys. 277 (1) (1999) $1-18$;

R.M.Yamaleev, "Elliptic and Hyperelliptic Deformed Mechanics in $n$-Dimesional Phase Space", JINR Communications,P2-94-109,Dubna, (1995);

R.M.Yamaleev, "Generalized Lorentz-Force Equations", Ann.Phys. 292 (1) (2001) 157-178.

[16] R.M.Yamaleev, Extended Relativistic Dynamics of Charged Spinning Particle in Quaternionic Formulation, Advances in Applied Clifford Algebras, 13(2), (2003), 183-218.

R.M.Yamaleev, Ternary Electrodynamics, Far East J.Dynamic Systems, 9(2) (2007) 307-324. 